\title{
DE FIESTA ANTES DE LA FIESTA: RELACIÓN ENTRE ESTA PRÁCTICA DE CONSUMO DE ALCOHOL CON LOS PROBLEMAS DERIVADOS DEL USO DE ALCOHOL EN JÓVENES ARGENTINOS
}

\section{ASSOCIATION BETWEEN ATTENDANCE TO PREGAMING EVENTS AND ALCOHOL-RELATED CONSEQUENCES IN ARGENTINEAN YOUTH}

Angelina Pilatti ${ }^{1}$, Paula Etkin ${ }^{1}$, Eugenia Urioste Parra ${ }^{1}$ y Ricardo Marcos Pautassi ${ }^{2}$

${ }^{1}$ Universidad Nacional de Córdoba, Argentina

${ }^{2}$ Instituto de Investigación Médica M. y M. Ferreyra

\section{Abstract}

Aims. This work described patterns of alcohol drinking in youth that attended pregaming events (i.e., the practice of drinking alcoholic beverages before attending a social/sporting event) and analyzed the relationship between frequency of pregaming, alcohol consumption at pregaming and alcohol-related negative consequences; and the modulation of these variables by the age of onset of alcohol drinking. Methods. 493 participants (62.3\% women) completed an online survey that determine the frequency of attendance at pregaming events, the quantity of alcohol consumed in those events, risky alcohol drinking, alcohol consumption during a typical drinking week or during the most intense drinking week, and the number of negative alcohol-related consequences. Results. There was a significant and positive correlation between frequency and quantity of alcohol consumed at pregaming with frequency of risky drinking, and with the number of negative alcohol-related consequences. The effect of frequency of attendance at pregaming events upon the number of negative alcoholrelated consequences was significant after controlling for the effect of the other independent variables. Conclusions. The results suggest that this consumption practice (i.e., pregaming) involves distinctive risks and represents a vulnerability factor for the development of negative alcohol-related consequences.

Keywords: pregaming, alcohol, negative consequences, youth.

\section{Resumen}

Objetivos. La previa es una práctica de consumo de alcohol que implica la ingesta de esta sustancia antes de asistir a un evento social, deportivo o musical donde puede, o no, consumirse más alcohol. En este trabajo describimos el perfil de consumo de alcohol de jóvenes asistentes a previas y analizamos la relación entre frecuencia de asistencia a previas y consumo de alcohol con la experimentación de consecuencias negativas, así como la modulación de estas variables por la edad de inicio de consumo. Método. 493 participantes (62.3\% mujeres) completaron una encuesta online para determinar la frecuencia de asistencia a previas y cantidad de consumo de alcohol en previas, consumo riesgoso de alcohol, consumo de alcohol durante una semana típica de consumo y durante la semana de consumo más intensa y consecuencias negativas del consumo de alcohol. Resultados. Se encontró una correlación significativa positiva entre frecuencia y cantidad de consumo de alcohol en previas con frecuencia de consumo riesgoso de alcohol y cantidad de problemas experimentados. El efecto de la frecuencia de asistencia a previas sobre la cantidad de consecuencias negativas fue significativo controlando el efecto de otras variables independientes. Conclusiones. Los jóvenes asistentes a previas exhiben un nivel de vulnerabilidad superior al que caracteriza a la población general de jóvenes para el desarrollo de un mayor número de problemas con el alcohol.

Palabras clave: prácticas de consumo excesivo de alcohol, consecuencias negativas, jóvenes. 
Argentina, a nivel poblacional, es el segundo país de América Latina con mayor consumo de alcohol per cápita (PAHO, 2015). Estudios realizados en este país de América del sur indican que el $80 \%$ de los adolescentes de 13 a 18 años (Pilatti, Godoy, Brussino, \& Pautassi, 2013) y el 93.1\% de jóvenes estudiantes universitarios (Pilatti, Caneto, Garimaldi, Vera, \& Pautassi, 2014) reportó tomar bebidas alcohólicas en el año previo; mientras que alrededor del 60\% de los jóvenes (Pilatti et al., 2014; Pilatti, Read, \& Caneto, 2016) presenta consumo episódico excesivo de alcohol (CEEA, usualmente binge drinking en la literatura anglosajona). Aunque hay variaciones, el CEEA es generalmente definido como el consumo de $\geq 4 / 5$ unidades (hombres/mujeres) en una misma ocasión de consumo (Wechsler, Davenport, Dowdall, Moeykens, \& Castillo, 1994) o en un periodo de, aproximadamente, dos horas (NIAAA, 2004). La NIAAA (2004) propuso una definición estandarizada de binge drinking como el patrón de consumo de alcohol que resulta en una concentración de alcohol en sangre de $\geq 0.08$ gramos por ciento. Esta misma entidad indicó que, en un adulto típico, esto equivale al consumo de $\geq 4 / 5$ unidades ( 1 unidad $\approx 14$ gramos) en aproximadamente dos horas. En el presente trabajo CEEA y consumo binge son considerados como sinónimos y se definen como el consumo de $\geq 4 / 5$ unidades (hombres/mujeres) en una misma ocasión de consumo.

Los jóvenes con CEEA experimentan un número significativamente mayor de problemas derivados del consumo de alcohol (por ejemplo, deterioro del sueño y la alimentación, aumento de comportamientos impulsivos, falta de control sobre cuánto alcohol se toma, desarrollo de tolerancia a los efectos del alcohol, episodios de amnesia, despertarse en un lugar inesperado e, incluso, que una persona cercana se haya preocupado por su nivel de consumo de alcohol) que los jóvenes bebedores sin este tipo de consumo (Keough, O'Connor, \& Read, 2016; Pilatti et al., 2016; Read, Beattie, Chamberlain, \& Merrill, 2008). Las consecuencias del CEEA pueden ser aún más groseras, e incluyen cambios estructurales del sistema nervioso central (Squeglia et al., 2012), que han sido confirmados mediante modelos animales (Vetreno, Yaxley, Paniagua, Johnson, \& Crews, 2017).

En la última década se observó un giro de la medición del consumo de alcohol en población general a mediciones en poblaciones o contextos específicos (e.g., en fines de semana; Finlay, Ram, Maggs, \& Caldwell, 2012). Una práctica, denominada prepartying o pregaming en la literatura anglosajona, es el consumo de alcohol antes de asistir a un evento social, deportivo o musical (Pedersen \& Labrie, 2007; White \& Hingson, 2014; Zamboanga et al., 2011). En el evento posterior se podrá, o no, consumir cantidades adicionales de alcohol. El prepartying es una práctica de alta prevalencia entre adolescentes (Kenney et al., 2010; Zamboanga et al., 2011) y jóvenes (Paves, Pedersen, Hummer, \& Labrie, 2012; Rutledge et al., 2016) de Estados Unidos, y en la misma se consumen elevadas cantidades de alcohol en un período corto de tiempo (Bachrach, Merrill, Bytschkow, \& Read, 2012; Haas, Smith, Kagan, \& Jacob, 2012; LaBrie, Hummer, Pedersen, Lac, \& Chithambo, 2012). Haas et al. (2012) encontraron que el $65 \%$ de una muestra de adolescentes, en la transición entre el nivel medio y la universidad, había realizado este tipo de práctica consumiendo, en promedio, tres unidades de alcohol ( 1 unidad $\approx 14$ gramos) en 27 minutos o menos. En este marco, no sorprende que esta práctica de consumo sea un importante predictor del consumo hasta la ebriedad (Borsari et al., 2007; Miller, Borsari, Fernandez, Yurasek, \& Hustad, 2016), de elevadas concentraciones de alcohol en sangre (Barnett, Orchowski, Read, \& Kahler, 2013; Barry, Stellefson, PiazzaGardner, Chaney, \& Dodd, 2013; Borsari et al., 2007) y de una mayor cantidad de consecuencias negativas (Paves et al., 2012).

En Argentina, país donde se llevó adelante el presente trabajo, el concepto que más se ajusta a esta práctica de consumo de alcohol es el denominado previa. Dentro de nuestro conocimiento, el único trabajo realizado en Argentina sobre este fenómeno indica que, usualmente, se considera a la previa como "una etapa dentro de la salida prevista (...) [que se caracteriza por] la ingesta de grandes cantidades de alcohol en un corto período de tiempo" (Del Zotto Libonati, 2015, pág 25). Así entonces, el concepto de previa es equiparable al de pregaming/prepartying (Pedersen \& Labrie, 2007; Zamboanga et al., 2011), entendiendo a ambas prácticas como el consumo de alcohol antes de asistir al evento de la salida (i.e. evento social, deportivo o musical) donde puede, o no, consumirse más alcohol.

Las investigaciones sobre sobre este tipo de prácticas se concentran en Norte-América y algunos países de Europa (Foster \& Ferguson, 2014), siendo aún 
escasos los trabajos realizados en Argentina. El único antecedente local que conocemos señala que un $82 \%$ de una muestra de estudiantes de nivel medio y estudiantes universitarios asistió, alguna vez en la vida, a previas y que el $68.5 \%$ de los asistentes a previas exhibió CEEA (Del Zotto Libonati, 2015). El presente trabajo describe el perfil de consumo de alcohol en una muestra de jóvenes argentinos, asistentes a previas, y analiza la relación entre la frecuencia de asistencia a previas con la experimentación de consecuencias negativas, así como la modulación de estas variables por la edad de inicio del consumo de alcohol. El contacto temprano con el alcohol es un factor de riesgo para exhibir problemas con el alcohol y otras sustancias (Hingson, Heeren, Winter, \& Wechsler, 2005). Así entonces, los objetivos específicos de este trabajo son: 1-describir indicadores de consumo de alcohol (frecuencia y cantidad usual, frecuencia de (EEA) en jóvenes argentinos asistentes a previas; 2-examinar posibles diferencias en el consumo de alcohol y en los indicadores sobre la previa en función del momento del inicio del consumo de alcohol; 3-analizar el efecto de la frecuencia de asistencia a previas sobre la cantidad de consecuencias negativas derivadas del uso de alcohol.

\section{Raft'tioiogntes}

Durante agosto de 2016 se difundió, a través de redes sociales y contactos de e-mail, la invitación al estudio, convocando a jóvenes (18 a 30 años) que hubiesen asistido a previas en el último año y tomado alcohol en el último mes. Se descartaron 18 casos por alguna de estas razones: sin consumo de alcohol en el último mes $(n=10)$, respuestas inconsistentes $(n=6)$ y ausencia de asistencia a previas en el último año $(n=2)$. La muestra final fue de 493 participantes $(62.3 \%$ [n=308] de mujeres; Media de edad de hombres y mujeres $=23.08 \pm 3.47$ y $22.08 \pm 2.94$, respectivamente) . Casi un tercio de la muestra se concentró en el rango de edad de $20-21$ (31.2\%), mientras que el resto se distribuyó entre 18-19 (17.1\%), 22-23 (19.7\%), $24-25$ (12.4\%), 26-27 (9.8\%) y 28-30 (9.8\%). El 87\% eran estudiantes universitarios $(81.5 \%$ de universidades públicas y $5.5 \%$ de universidades privadas, porcentaje que reproduce la distribución del sistema universitario argentino) y el $4.3 \%$ eran estudiantes de nivel terciario. La encuesta empleada fue de modalidad online, por lo que se controló la potencial duplicación de participación chequeando el e-mail o número de teléfono provisto por los participantes al finalizar la encuesta (ver procedimiento).

\section{Procedimiento}

Los datos se recolectaron mediante una encuesta online desarrollada con el software LimeSurvey (licencia oficial de la Universidad Nacional de Córdoba). La invitación contenía un link a la encuesta que incluía el formulario de consentimiento informado. Al presionar el botón continuar, al final del formulario de consentimiento, los participantes indicaban su consentimiento a formar parte del estudio. Los participantes recibían avisos por cada dato sin completar para evitar los datos ausentes. Se garantizó el manejo confidencial de los datos. En la invitación se indicaba que, entre aquellos que completaran la encuesta, se sortearía una estadía en un centro turístico. Aunque no se solicitó información que permita identificar a los participantes (i.e., nombre y apellido), se invitó a los participantes a dejar su dirección de correo electrónico y/o número de teléfono para ser contactados en caso que resulten ganadores del sorteo. Estos datos fueron también utilizados para pesquisar la potencial duplicación de participación.

\section{Instrumentos}

Indicadores sobre la previa: Se definió (i.e., consumir alcohol antes de asistir a un evento social, deportivo o musical donde puede, o no, consumirse más alcohol) y midió el comportamiento relacionado a previas siguiendo estudios previos (Bachrach et al., 2012; LaBrie et al., 2012). Específicamente, se midió la frecuencia de asistencia a previas en los últimos doce meses (desde $0=$ no asistí a previas en los últimos 12 meses hasta $7=20$ más veces por semana) y en los últimos 30 días (i.e., cantidad de previas). Se midió la cantidad de unidades de alcohol consumida, en promedio, durante las previas. Una imagen informaba qué volumen de alcohol, para una variedad de bebidas, correspondía a una medida de alcohol. Diferentes países presentan variaciones en la definición operacional de una unidad estándar de alcohol (Dawson, 2003), sin embargo la NIAAA (2004) la define como conteniendo 14 gramos de alcohol. En este trabajo usamos la definición operacional de unidad estándar de alcohol propuesta por la NIAAA, ya que es una de las más ampliamente utilizadas en la literatura. 
Cuestionario de Consumo de Alcohol: se midió la cantidad de unidades de alcohol consumidas en cada día (lunes a domingo) de una semana típica de consumo de alcohol y de la semana de consumo más intenso de alcohol durante los 30 días previos. Además, los participantes indicaron con qué frecuencia (desde $0=n o$ tomé esa bebida en los últimos 30 días hasta $8=$ casi todos los días) habían consumido una variedad de 15 bebidas alcohólicas (cerveza, fernet, vodka, vino, ron, entre otras) durante los 30 días previos a la encuesta. Se midió la frecuencia (desde $0=$ nunca hasta $7=\geq 4$ veces por semana) de CEEA (>4/5 unidades de alcohol en mujeres/hombres durante una misma ocasión de consumo) y de consumo hasta la ebriedad (desde $0=$ nunca me emborraché/no me emborraché en los últimos 30 días hasta 9=nueve veces o más) durante los 30 días previos.

Versión en español del Cuestionario de Consecuencias del Consumo de Alcohol en Jóvenes Adultos: utilizamos la adaptación al español (Pilatti et al., 2016) del Young Adult Alcohol Consequences Questionnaire (Read, Kahler, Strong, \& Colder, 2006). Este instrumento mide 48 consecuencias negativas en ocho sub-escalas (Autopercepción, Relaciones Sociales-Interpersonales, Comportamientos Riesgosos, Amnesia/Desmayos, Déficit en el Control de Impulsos, Dependencia Psicológica, Desempeño Académico/Ocupacional y Cuidado Personal (confiabilidad entre $\alpha=.70$ y $\alpha=.91$; Pilatti et al., 2016). Los participantes indicaron si habían experimentado (si/no) cada consecuencia en los últimos 30 días.

Edad de inicio del consumo: Se clasificó a los bebedores tempranos y tardíos en función de la edad del primer consumo de $\geq 1$ medida de alcohol. Específicamente, aquellos que reportaron consumir alcohol $\leq 14$ años fueron clasificados como bebedores tempranos (IT+) y aquellos que comenzaron a los $\geq 15$ como bebedores tardíos (IT-). Estos puntos de corte fueron establecidos en función de estudios previos (Lee, Young-Wolff, Kendlern \& Prescott, 2012).

\section{Análisis de datos}

Utilizamos estadística descriptiva para describir la ocurrencia de asistencia a previas y el perfil de consumo de alcohol, para la muestra total y en función del sexo y de la edad de inicio del consumo de alcohol. Para determinar diferencias en el uso de alcohol y en la asistencia a previas entre varones y mujeres, y entre IT+ e IT-, se utilizó el estadístico Chi Cuadrado o la prueba $t$ de Student (variables nominales y continuas, respectivamente). Luego, se correlacionaron las conductas de previas (frecuencia de asistencia en el último mes o último año y el promedio de consumo de alcohol en previas) con la sumatoria total de consecuencias negativas, la frecuencia de CEEA y consumo hasta la ebriedad, y la cantidad de consumo de alcohol en cada día de una semana típica y de una semana de consumo más intenso. Se empleó el coeficiente de correlación de Spearman porque algunas de las variables no presentaron valores adecuados de asimetría y curtosis. Mediante una regresión jerárquica analizamos si la frecuencia de asistencia a previas explicaba la mayor cantidad de consecuencias negativas, controlando el efecto del total de alcohol consumido en una semana típica (sumatoria de las medidas consumidas en cada día de una semana típica de consumo de alcohol), la frecuencia de CEEA y la edad de inicio del consumo de alcohol. Asimismo, y debido a las variaciones en el consumo de alcohol entre hombres y mujeres, se controló en la regresión el efecto del sexo. El error tipo I fue fijado en 0.05

Los valores descriptivos y las notaciones estadísticas (i.e., valores de $\chi^{2}$, valores de $F$, valores de $t$ de Student, valores de $p$, etc.) para la mayoría de los análisis inferenciales se presentan en las tablas.

\section{RESULTADOS}

\section{Resultados descriptivos}

Frecuencia de asistencia a previas y consumo de alcohol en previas

El 46\% de los participantes reportó haber asistido, en el último año, al menos 2-3 veces por mes a previas. Durante el mes previo asistieron, en promedio, a $2.76 \pm 2.18$ previas donde consumieron una media de $5.59 \pm 3.42$ medidas de alcohol ( $\approx 78.26$ gramos) en cada evento (véase Tabla 1).

\section{Bebidas más consumidas en el mes previo}

Cerveza (87.2\%), fernet (72\%) y vino (63.9\%) fueron las bebidas consumidas por un mayor número de participantes y con mayor frecuencia (i.e., mayor cantidad de días en el mes). Puntualmente, el 54.5\%, 34.9\% y 31\% indicó tomar estas bebidas $\geq 1$ vez por semana (véase Tabla 2). 
Tabla 1. Frecuencia de asistencia a previas y cantidad de alcohol consumida en previas, consumo episódico excesivo y consumo hasta la ebriedad para la muestra total y en función del sexo y la edad de inicio del consumo de alcohol

\begin{tabular}{|c|c|c|c|c|c|c|c|}
\hline & Total & Mujeres & Hombres & $t / \chi^{2}$ & IT+ & IT- & $t$ \\
\hline \multicolumn{8}{|l|}{ F Prev $12 \mathrm{M}$} \\
\hline $1-4$ en $12 M$ & 14.8 & 16.6 & 11.9 & & 9.3 & 17.5 & \\
\hline 5-8 en $12 M$ & 13.2 & 14.3 & 11.4 & & 11.8 & 13.9 & \\
\hline $9-11$ en $12 \mathrm{M}$ & 10.1 & 12.0 & 7.0 & & 8.1 & 11.1 & \\
\hline 1 por $\mathrm{M}$ & 15.4 & 14.6 & 16.8 & & 14.3 & 16.0 & \\
\hline 2-3 por $\mathrm{M}$ & 29.3 & 29.5 & 29.2 & & 29.8 & 29.2 & \\
\hline 1 por $\mathrm{S}$ & 12.8 & 9.1 & 18.9 & & 19.9 & 9.3 & \\
\hline 2 o más por 5 & 4.3 & 3.9 & 4.9 & & 6.8 & 3.0 & \\
\hline Prev 30 Días & $2.76 \pm 2.18$ & $2.64 \pm 2.12$ & $2.98 \pm 2.27$ & 1.69 & $3.42 \pm 2.46$ & $2.45 \pm 1.95$ & $4.47^{* \star *}$ \\
\hline Med Prev & $5.59 \pm 3.42$ & $4.84 \pm 2.89$ & $6.83 \pm 3.84$ & $6.50^{* * *}$ & $6.58 \pm 3.84$ & $5.11 \pm 3.08$ & $4.57^{\star \star *}$ \\
\hline \multicolumn{8}{|l|}{ F CEEA } \\
\hline Sin CEEA & 21.1 & 22.4 & 18.9 & ${ }^{1} .84$ & 14.3 & 24.4 & ${ }^{2} 6.66^{* *}$ \\
\hline 1 en $M$ & 18.7 & 21.4 & 14.1 & & 7.5 & 24.1 & \\
\hline 2 en $M$ & 17.4 & 17.5 & 17.3 & & 14.3 & 19 & \\
\hline 3 en $M$ & 11 & 11.7 & 9.7 & & 15.5 & 8.7 & \\
\hline 1 por $S$ & 16.8 & 14.3 & 21.1 & & 22.4 & 14.2 & \\
\hline 2 por $S$ & 9.7 & 7.5 & 13.5 & & 14.9 & 7.2 & \\
\hline 3 por $S$ & 3.4 & 3.2 & 3.8 & & 6.2 & 2.1 & \\
\hline 4 por $S$ & 1.8 & 1.9 & 1.6 & & 5 & .3 & \\
\hline CEEB & $.99 \pm 1.47$ & $.78 \pm 1.14$ & $1.35 \pm 1.85$ & $4.26^{* * *}$ & $1.48 \pm 1.92$ & $.76 \pm 1.12$ & $5.23^{* * *}$ \\
\hline
\end{tabular}

Nota: Los resultados se presentan como porcentajes de casos que caen dentro de cada categoría. Para las variables continuas, los datos se presentan como medias y desviación estándar en cada categoría. IT+ = inicio temprano; IT- = inicio tardío; F Prev 12 M = frecuencia de asistencia a previas en último año; 12 M = año; $\mathrm{M}$ = mes; $\mathrm{S}$ = semana; Prev 30 Días = cantidad de días con asistencia a previas en el último mes; Med Prev = promedio de medidas de alcohol consumida en cada previa. F CEEA = frecuencia de consumo episódico excesivo; C E EB = cantidad de episodios de consumo hasta la ebriedad.

${ }^{1}$ Asociación entre sexo y ocurrencia de CEEA.

${ }^{2}$ Asociación entre momento de inicio del consumo y ocurrencia de CEEA.

Tabla 2. Frecuencia de consumo de una variedad de bebidas alcohólicas durante los 30 días previos

\begin{tabular}{|c|c|c|c|c|c|c|c|c|c|c|c|c|c|c|c|}
\hline & Cerveza & Fernet & Vino & Vodka & V/Energ & Aperitivo & Ron & Gancia & Whiskey & Licor & Tequila & BM L & Ginebra & L Menta & Sidra \\
\hline Sin consumo & 12.8 & 28.0 & 36.1 & 64.3 & 60.4 & 68.4 & 83.4 & 80.9 & 87.4 & 87.4 & 92.7 & 85.2 & 91.7 & 94.1 & 96.8 \\
\hline $1 \mathrm{M}$ & 8.7 & 10.1 & 15.0 & 11.6 & 14.4 & 15.4 & 7.5 & 7.1 & 5.1 & 5.9 & 3.7 & 7.7 & 5.3 & 2.0 & 1.8 \\
\hline $2 \mathrm{M}$ & 9.1 & 13.8 & 9.9 & 6.7 & 6.1 & 7.1 & 2.8 & 4.1 & 2.8 & 3.0 & 1.0 & 2.4 & .8 & 1.0 & .6 \\
\hline $3 \mathrm{M}$ & 14.8 & 13.2 & 7.9 & 6.9 & 6.1 & 3.7 & 2.0 & 3.2 & .6 & 1.0 & .6 & 1.0 & .6 & .8 & .2 \\
\hline 1 por $s$ & 17.2 & 16.6 & 16.4 & 6.9 & 7.3 & 3.9 & 2.8 & 2.6 & 2.0 & 1.2 & 1.2 & 1.0 & .6 & .8 & .2 \\
\hline 2 por $s$ & 16.8 & 12.2 & 7.5 & 1.8 & 4.3 & .8 & .4 & 1.6 & 1.0 & .6 & .4 & 1.0 & .4 & .6 & 0 \\
\hline 3 por $s$ & 9.5 & 4.5 & 4.9 & 1.2 & 1.0 & .4 & .8 & .4 & .8 & .6 & .2 & 1.4 & .4 & .4 & .2 \\
\hline 4 por $S$ & 5.7 & 1.0 & 1.2 & .6 & .4 & .4 & .2 & 0 & 0 & 0 & .2 & 0 & 0 & .2 & .2 \\
\hline Casi todos los días & 5.3 & .6 & 1.0 & 0 & 0 & 0 & 0 & 0 & .2 & .2 & 0 & .2 & .2 & 0 & 0 \\
\hline
\end{tabular}

Nota: Los resultados se presentan como porcentajes de casos que caen dentro de cada categoría. Sin consumo = sin consumo de esa bebida; $\mathrm{M}=$ mes; $\mathrm{S}=$ semana; V/Energ = vodka con energizante; $B M L$ = Bebida alcohólica mixta con jugo de limón; Aperitivo = aperitivo de sabor amargo; L Menta = licor a base de hierbas, especias y aceite esencial de menta 
Consumo de alcohol en una semana típica de consumo de alcohol y en la semana de mayor consumo

La gran mayoría de los participantes reportó, en una semana típica de consumo de alcohol, no consumir alcohol entre el domingo y el miércoles, pero sí hacerlo los días viernes y sábado. La cantidad de medidas de alcohol consumidas por los bebedores fue mayor durante estos últimos días que durante el resto de los días de la semana. En la semana de consumo más intenso de alcohol aumentó de manera marcada, respecto a la semana típica, la cantidad de participantes que reportó consumir alcohol de lunes a jueves. Como era de esperar, también aumentó la media de medidas de alcohol consumidas en cada día de la semana. Estos resultados se presentan en la Tabla 3.

Tabla 3. Consumo de alcohol en una semana típica y en la semana de consumo más intenso para la muestra total y en función del sexo y la edad de inicio del consumo de alcohol

\begin{tabular}{|c|c|c|c|c|c|c|c|c|c|}
\hline & & & Bebed & & & & & & \\
\hline & Rango M & Medidas & Total & Mujeres & Hombres & $\chi^{2}$ & IT+ & IT- & $\chi^{2}$ \\
\hline \multicolumn{10}{|l|}{ ST } \\
\hline Lunes & $1-4$ & $1.75 \pm 1.02$ & 4.1 & 2.6 & 6.5 & $4.49^{*}$ & 8.7 & 1.8 & $13.22^{\star \star \star}$ \\
\hline Martes & $1-4$ & $1.79 \pm 1.11$ & 5.9 & 4.5 & 8.1 & 1.65 & 9.3 & 4.2 & $5.09^{*}$ \\
\hline Miércoles & $1-6$ & $1.89 \pm 1.16$ & 15.4 & 12 & 21.1 & $7.29^{* *}$ & 20.5 & 13 & $4.73^{*}$ \\
\hline Jueves & $1-16$ & $2.80 \pm 2.43$ & 23.5 & 18.8 & 31.4 & $10.07^{* *}$ & 34.8 & 18.1 & $16.83^{* * *}$ \\
\hline Viernes & $1-16$ & $3.54 \pm 2.80$ & 68.6 & 62.3 & 78.9 & $14.74^{* * *}$ & 73.3 & 66.3 & 2.48 \\
\hline Sábado & $1-30$ & $4.33 \pm 3.32$ & 86.6 & 84.7 & 89.7 & 2.48 & 91.3 & 84.3 & $4.54^{*}$ \\
\hline Domingo & $1-6$ & $1.89 \pm 1.17$ & 16.6 & 11 & 25.9 & $18.53^{* \star *}$ & 21.7 & 14.2 & $4.50^{*}$ \\
\hline \multicolumn{10}{|l|}{ SI } \\
\hline Lunes & $1-16$ & $3.36 \pm 3.42$ & 15.2 & 12 & 20.5 & $6.52^{* *}$ & 24.2 & 10.8 & $15.05^{\star * *}$ \\
\hline Martes & $1-16$ & $3.56 \pm 2.98$ & 20.5 & 17.2 & 25.9 & $5.42^{*}$ & 29.8 & 16 & $12.77^{\star \star \star}$ \\
\hline Miércoles & $1-20$ & $3.85 \pm 3.32$ & 34.7 & 31.8 & 39.5 & 2.98 & 44.7 & 29.8 & $10.63^{\star * *}$ \\
\hline Jueves & $1-22$ & $4.36 \pm 3.73$ & 44.2 & 38.6 & 53.5 & $10.37^{* * *}$ & 55.3 & 38.9 & $11.86^{* * *}$ \\
\hline Viernes & $1-30$ & $4.90 \pm 4.07$ & 78.9 & 74.7 & 85.9 & $8.82^{* *}$ & 83.9 & 76.5 & 3.51 \\
\hline Sábado & $1-30$ & $5.69 \pm 4.12$ & 86.4 & 87.7 & 84.3 & 1.10 & 87.6 & 85.8 & .28 \\
\hline Domingo & $1-16$ & $3.32 \pm 3.13$ & 23.7 & 17.9 & 33.5 & $15.65^{* * *}$ & 31.7 & 19.9 & $8.34^{* *}$ \\
\hline
\end{tabular}

Nota: Los resultados se presentan como porcentajes de casos que caen dentro de cada categoría. Para las variables continuas, los datos se presentan como medias y desviación estándar en cada categoría. ST = semana típica de consumo de alcohol, SI = semana de consumo más intenso de alcohol; Rango M = rango de unidades estándar de alcohol consumidas entre los bebedores; Medidas = media de medidas consumidas calculada a partir de los que reportaron consumir; Bebedores = participantes que reportaron tomar alcohol cada día; IT+ = inicio temprano; IT- = inicio tardío

Consumo episódico excesivo de alcohol y consumo hasta la ebriedad

El 78.9\% de los participantes indicó haber tenido $\geq 1$ episodio de CEEA en los últimos 30 días, y el 31.7\% indicó consumir de manera excesiva al menos una vez a la semana. La media de episodios de consumo hasta la ebriedad en los últimos 30 días fue .99+1.47. Estos resultados se presentan en la Tabla 1.

\section{Problemas asociados al uso de alcohol}

Los participantes experimentaron una media de $11.73 \pm 8.53$ consecuencias negativas por su consumo de alcohol. La Tabla 4 presenta la ocurrencia de cada una de las consecuencias negativas para la muestra total y en función del sexo y del momento del inicio del consumo de alcohol. 
Tabla 4. Ocurrencia de consecuencias derivadas del uso de alcohol para la muestra total y en función del sexo y del momento del inicio del consumo

\begin{tabular}{|c|c|c|c|c|c|c|c|}
\hline & Total & Mujeres & Hombres & $t$ & IT+ & IT- & $t$ \\
\hline He tenido una resaca & 75.1 & 72.9 & 78.7 & & 80.0 & 72.6 & \\
\hline Tomé más alcohol de lo que había planeado & 69.9 & 66.3 & 75.9 & & 77.4 & 66.1 & \\
\hline He dicho o hecho cosas vergonzosas & 59.8 & 60.8 & 58.0 & & 61.9 & 58.7 & \\
\hline No podía recordar una parte de la noche anterior. & 55.4 & 53.3 & 59.0 & & 61.9 & 52.1 & \\
\hline Me he sentido muy descompuesto o he vomitado & 48.2 & 48.1 & 48.3 & & 54.2 & 45.2 & \\
\hline He hecho cosas de manera impulsiva que luego lamenté & 47.3 & 46.0 & 49.4 & & 54.2 & 43.9 & \\
\hline No he sido capaz de recordar largos espacios de tiempo & 39.8 & 39.5 & 40.2 & & 41.9 & 38.7 & \\
\hline No he dormido adecuadamente. & 39.8 & 35.1 & 47.7 & & 41.3 & 39.0 & \\
\hline He dicho cosas que después lamenté. & 39.8 & 41.6 & 36.8 & & 46.5 & 36.5 & \\
\hline Necesitaba más cantidad de alcohol para sentir algún efecto & 34.6 & 33.0 & 37.4 & & 43.2 & 30.3 & \\
\hline No he comido de manera apropiada & 33.5 & 28.5 & 42.0 & & 36.1 & 32.3 & \\
\hline He estado menos activo físicamente & 33.3 & 28.9 & 40.8 & & 35.5 & 32.3 & \\
\hline Me he sentido mal conmigo mismo & 31.4 & 30.6 & 32.8 & & 36.1 & 29.0 & \\
\hline He tenido "olvidos" & 29.7 & 26.8 & 34.5 & & 31.6 & 28.7 & \\
\hline He tomado riesgos estúpidos & 29.7 & 27.5 & 33.3 & & 38.1 & 25.5 & \\
\hline Me he sentido con menos energía o cansado & 29.2 & 23.0 & 39.7 & & 28.4 & 29.7 & \\
\hline He encontrado difícil limitar cuánto alcohol consumo. & 28.0 & 20.6 & 40.2 & & 36.1 & 23.9 & \\
\hline Me he sentido triste o algo deprimido & 27.3 & 27.8 & 26.4 & & 25.8 & 28.1 & \\
\hline Hice comentarios crueles o hirientes a alguien & 24.9 & 22.7 & 28.7 & & 34.2 & 20.3 & \\
\hline Me he sentido culpable & 23.2 & 23.0 & 23.6 & & 20.6 & 24.5 & \\
\hline He intentado dejar de tomar porque estaba tomando mucho. & 21.5 & 18.2 & 27.0 & & 23.9 & 20.3 & \\
\hline He pasado demasiado tiempo tomando alcohol & 20.4 & 13.7 & 31.6 & & 32.9 & 14.2 & \\
\hline Me he vuelto grosero, pesado, o he insultado & 20.0 & 17.9 & 23.6 & & 25.8 & 17.1 & \\
\hline Familiares se han quejado acerca de mi consumo & 18.9 & 13.7 & 27.6 & & 23.9 & 16.5 & \\
\hline He aumentado de peso debido a mi consumo de alcohol & 18.9 & 16.8 & 22.4 & & 23.2 & 16.8 & \\
\hline Me he sentido decepcionado & 18.7 & 18.2 & 19.5 & & 18.1 & 19.0 & \\
\hline No he estado tan ágil mentalmente & 16.6 & 13.7 & 21.3 & & 18.7 & 15.5 & \\
\hline Descuidé obligaciones familiares, laborales o de estudio & 16.3 & 10.3 & 26.4 & & 25.8 & 11.6 & \\
\hline He faltado al trabajo o a clases por una resaca & 16.3 & 12.7 & 22.4 & & 20.6 & 14.2 & \\
\hline Mi apariencia física se ha visto deteriorada & 15.5 & 12.4 & 20.7 & & 20.6 & 12.9 & \\
\hline He tenido problemas con familiares cercanos. & 14.0 & 11.7 & 17.8 & & 17.4 & 12.3 & \\
\hline La calidad de mi trabajo o estudio ha disminuido & 13.8 & 10.3 & 19.5 & & 13.5 & 13.9 & \\
\hline He tenido relaciones sexuales que después lamenté & 13.1 & 10.0 & 18.4 & & 14.8 & 12.3 & \\
\hline No me cuidé de ETS o de la posibilidad de embarazo & 11.4 & 10.0 & 13.8 & & 12.9 & 10.6 & \\
\hline He realizado algún acto de vandalismo & 11.4 & 3.8 & 24.1 & & 19.4 & 7.4 & \\
\hline He manejado un auto & 11.4 & 5.2 & 21.8 & & 16.8 & 8.7 & \\
\hline
\end{tabular}


Tabla 4. Ocurrencia de consecuencias derivadas del uso de alcohol para la muestra total y en función del sexo y del momento del inicio del consumo (Continuación)

\begin{tabular}{|c|c|c|c|c|c|c|}
\hline Me he despertado en un lugar inesperado & 9.9 & 5.8 & 16.7 & 12.3 & 8.7 & \\
\hline He tenido menos tiempo para realizar mis actividades & 8.0 & 5.2 & 12.6 & 7.1 & 8.4 & \\
\hline Me he metido en peleas físicas & 5.6 & 1.0 & 13.2 & 9.0 & 3.9 & \\
\hline He tenido temblores al detener o reducir mi consumo & 5.2 & 3.1 & 8.6 & 7.1 & 4.2 & \\
\hline He lastimado a alguien mientras tomaba alcohol o estaba intoxicado & 4.7 & 1.0 & 10.9 & 7.1 & 3.5 & \\
\hline He tenido una nota más baja que lo usual en un examen & 4.3 & 2.1 & 8.0 & 5.8 & 3.5 & \\
\hline Me metí en problemas en el trabajo o en mis estudios & 3.9 & 2.7 & 5.8 & 6.5 & 2.6 & \\
\hline Me he desmayado & 3.7 & 2.7 & 5.2 & 3.9 & 3.5 & \\
\hline Me he sentido ansioso al disminuir mi consumo & 3.0 & 2.4 & 4.0 & 3.9 & 2.6 & \\
\hline He sentido que necesitaba tomar alcohol al levantarme & 2.8 & 2.1 & 4.0 & 3.2 & 2.6 & \\
\hline PA Total & $11.73 \pm 8.53$ & $10.41 \pm 7.92$ & $13.94 \pm 9.074 .39^{* * *}$ & $12.80 \pm 8.53$ & $9.92 \pm 8.24$ & $3.56^{* * *}$ \\
\hline
\end{tabular}

Nota: Los resultados se presentan como porcentajes de casos que caen dentro de cada categoría. Para las variables continuas, los datos se presentan como medias y desviación estándar en cada categoría. IT+ = inicio temprano; IT- = inicio tardío. Por cuestiones de espacio, la descripción de cada ítem está resumida

\section{Diferencias de grupo}

\section{Diferencias en función del sexo}

Los hombres, comparados con las mujeres, tomaron significativamente más alcohol en las previas aunque no se observaron diferencias estadísticamente significativas en la cantidad de veces que asistieron a previas en el último mes (ver Tabla 1). Los hombres presentaron una ocurrencia de consumo de alcohol significativamente mayor que las mujeres en la mayor parte de los días de una semana típica e intensa (ver Tabla 3). La ocurrencia de CEEA, pero no del consumo hasta la ebriedad (Tabla 1) y de problemas derivados del uso de alcohol (Tabla 4), fue estadísticamente similar en hombres y mujeres.

Diferencias en función de la edad de inicio del consumo de alcohol

Los jóvenes con IT+, comparados con aquellos con IT-, asistieron significativamente a mayor cantidad de previas, tomaron significativamente más alcohol y presentaron una ocurrencia de consumo de alcohol significativamente mayor en casi todos los días de las semanas típica e intensa (ver Tablas 1 y 3). El porcentaje de IT+ con CEEA fue significativamente más alto que entre los IT-. Los IT+ presentaron significativamente más episodios de consumo hasta la ebriedad y experimentaron mayor cantidad de consecuencias negativas que sus pares IT-.

\section{Análisis bivariados}

Indicadores sobre la previa y consumo de alcohol durante las semanas típica e intensa

Los resultados bivariados se presentan en la Tabla 5. Se observó una correlación significativa y positiva entre la frecuencia de asistencia a previas y la cantidad de alcohol consumida en todos los días de la semana de consumo intenso y en la mayoría de los días de una semana típica. La cantidad de alcohol consumida en cada previa correlacionó significativa y positivamente con la cantidad de alcohol consumida en todos los días de ambas semanas.

Indicadores sobre la previa, consumo episódico excesivo de alcohol, consumo hasta la ebriedad y problemas asociados al consumo

Los jóvenes que asistieron con mayor frecuencia a previas, y que allí consumieron mayor cantidad de alcohol, presentaron significativamente mayor frecuencia de CEEA, mayor cantidad de episodios de consumo hasta la ebriedad y mayor cantidad de problemas derivados del uso de alcohol (i.e., puntuaciones más altas en la mayoría de las sub-escalas del YAACQ). 
Tabla 5. Correlaciones entre los indicadores sobre las previas (frecuencia de asistencia y cantidad de consumo de alcohol) con el consumo durante una semana típica e intensa, consumo episódico excesivo de alcohol, consumo hasta la ebriedad y problemas derivados del consumo de alcohol

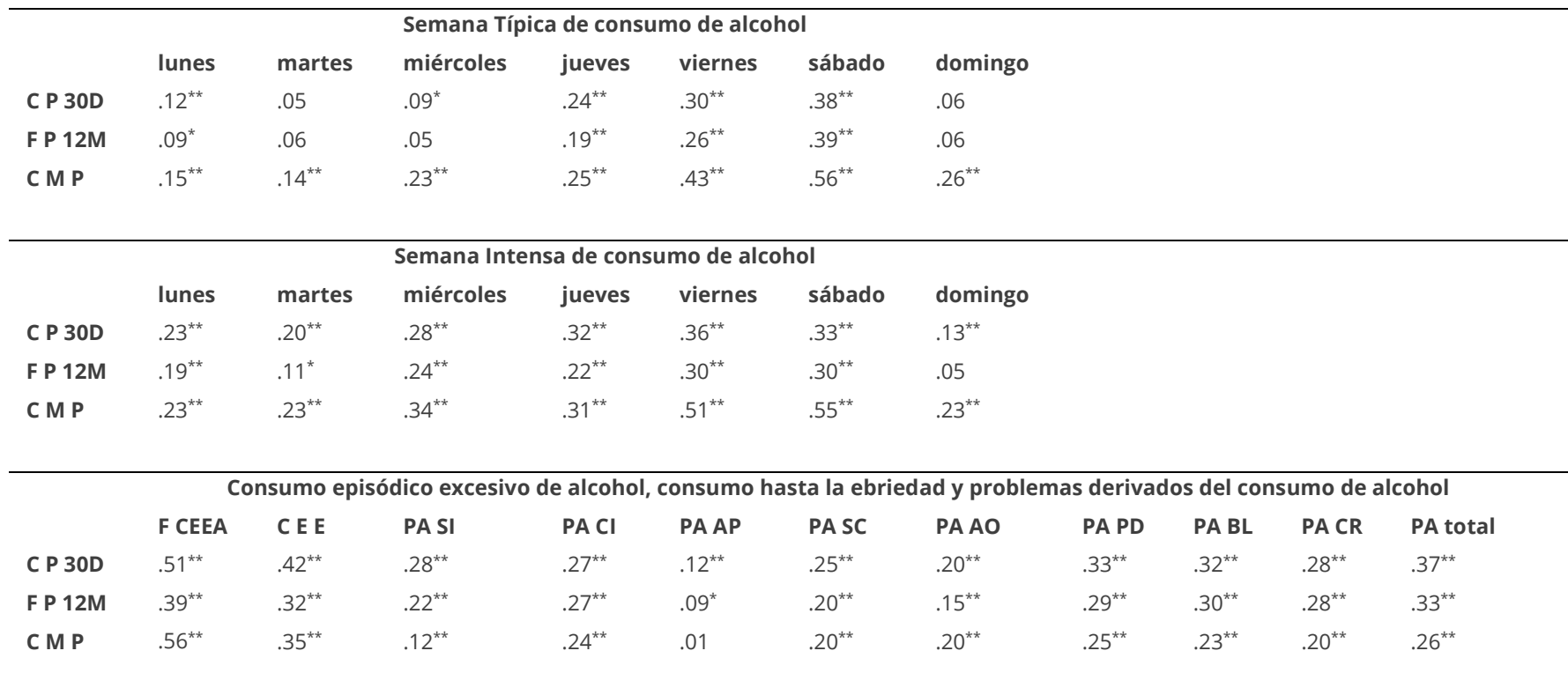

Nota: C P 30D = cantidad de previas en último mes; F P 12M = frecuencia de asistencia a previas en último año; C M P= cantidad de unidades de alcohol consumida por previa. F CEEA = frecuencia consumo episódico elevado de alcohol; C E E = cantidad de episodios de consumo hasta la ebriedad; PA= escalas del cuestionario de problemas con el alcohol: PA SI= Relaciones Sociales Interpersonales; PA CI = Déficit en el Control de Impulsos; PA AP = Autopercepción; $\mathrm{PACP}=$ Cuidado Personal; $\mathrm{PA} A O=$ Desempeño Académico/Ocupacional; $\mathrm{PA} D \mathrm{DP}=$ Dependencia Psicológica; $\mathrm{PA} A D=A m n e s i a / D e s m a y o s ; \mathrm{PA} C R=$ Comportamientos Riesgosos; PA total = puntaje total.

\section{Regresión múltiple}

En el primer paso, el sexo de los participantes y la edad de inicio del consumo de alcohol explicaron un $7 \%$ de la varianza de la cantidad de problemas derivados del uso de alcohol [F cambio $\left._{(2,461)}=17.37 p \leq .001\right]$. Ser hombre $(\beta=.18, t=3.95, p \leq .001)$ y tener una edad de inicio de consumo más baja ( $\beta=-.17, t=-3.862, p \leq .001)$ se asociaron a un mayor número de problemas por el uso de alcohol. El ingreso, en el segundo paso, del volumen de alcohol consumido durante una semana típica de consumo y la frecuencia de CEEA en el último mes aumentó un 7\% la varianza explicada de la variable dependiente [F cambio $(2$, 459)=19.92 $p \leq .001]$. Una mayor cantidad de alcohol consumida $(\beta=.14, t=2.23, p \leq .05)$ y una mayor frecuencia de $\operatorname{CEEA}(\beta=.18, t=2.87, p \leq .01)$ explicaron una mayor cantidad de problemas experimentados por el uso de alcohol. Asimismo, el efecto del momento del inicio del consumo dejó de ser significativo, indicando una mediación total del efecto de esta variable por aquellas incluidas en este paso. En el tercer paso, el ingreso de la frecuencia de asistencia a previas en el mes anterior incrementó la varianza explicada al 17\% [F cambio $\left._{(1,458)}=12.58 p \leq .001\right]$. Una mayor asistencia a previas se asoció a más problemas inducidos por el alcohol $(\beta=.18, t=3.559, p \leq .001)$. Los efectos del volumen de alcohol consumido en una semana típica y de la frecuencia de CEEA dejaron de ser significativos, sugiriendo que estos efectos están mediados por la frecuencia de asistencia a previas.

\section{DISCUSIÓN}

En este trabajo describimos el consumo de alcohol, y consecuencias asociadas al mismo, en jóvenes argentinos asistentes a previas, una práctica de consumo de alcohol comparable al prepartying o pregaming descripto en la literatura anglosajona. La frecuencia de asistencia a previas fue similar a la encontrada con estudiantes de nivel medio (Zamboanga et al., 2011) pero algo más baja que con estudiantes universitarios (Hustad et al., 2014; LaBrie et al., 2012; Merrill, Vermont, Bachrach, \& Read, 2013) de Estados Unidos. En consonancia con otros trabajos (LaBrie et al., 2012; Merrill et al., 2013) esta práctica de consumo se caracterizó por una media de consumo de alcohol elevada, incluso superior a la encontrada en muestras de población similar provenientes de Estados Unidos (LaBrie et al., 2012; Merrill et al., 2013). 
Todos los participantes de este estudio asistieron a previas durante el año previo, por lo que no es posible describir perfiles diferenciales de consumo de alcohol, y de problemas experimentados, en función de asistir o no a previas. La comparación con estudios previos, sin embargo, indica que la media de $\approx 12$ problemas por el uso de alcohol es superior a la encontrada entre jóvenes bebedores del medio local (Pilatti et al., 2014) pero similar a la encontrada entre asistentes a previas de Estados Unidos (Borsari et al., 2007; Merrill et al., 2013). La frecuencia de asistencia y el consumo de alcohol durante la previa se asociaron positiva y significativamente a un mayor consumo de alcohol y a una mayor cantidad de consecuencias negativas derivadas del uso de alcohol. Es posible que este resultado refleje la conocida relación entre el CEEA (característico de las previas) y los problemas con el alcohol (White \& Hingson, 2014). Sin embargo, los resultados de la regresión jerárquica indicaron que la frecuencia de asistencia a previas tiene un efecto único e independiente sobre los problemas asociados al uso de alcohol, que se mantiene aun controlando por el volumen de alcohol consumido y la frecuencia de CEEA. Esto concuerda con lo descripto por Labrie et al. (2016), quienes observaron que la frecuencia de asistencia a previas fue un mejor predictor de las consecuencias negativas asociadas al consumo del alcohol que el volumen total de alcohol consumido o que la frecuencia de CEEA. A diferencia de Haas et al. (2012), la frecuencia de asistencia a previas medió completamente el efecto de los indicadores de volumen y frecuencia de CEEA. En el estudio de Haas et al. (2012) los participantes eran bebedores de alcohol pero no todos eran asistentes a previas. Es posible, entonces, que para la sub-población específica del presente trabajo, caracterizada por un elevado consumo de alcohol y de problemas con el alcohol, se genere un "efecto techo" respecto a los indicadores de consumo de alcohol, que impide su expresión.

En conjunto, los resultados de Labrie et al. (2016) y los del presente estudio, rechazan la hipótesis que la frecuencia de asistencias a previas, y el consumo elevado de alcohol en las mismas, sea meramente un correlato, síntoma o conducta co-adjuntiva de aquellos que ya exhiben patrones de consumo riesgoso. Por el contrario, nuestros datos apoyan la noción que la asistencia a previas tendría un rol propio, promotor, en el involucramiento de los jóvenes en trayectorias de consumo riesgoso. Es decir, los resultados sugieren que la vulnerabilidad para el desarrollo de problemas con el alcohol es mayor en esta sub-población específica, la de jóvenes asistentes a previas, que en la población general de jóvenes (White \& Hingson, 2014). En los últimos años se ha intentado medir estilos de consumo de alcohol, y de otras sustancias, distintivos de prácticas de consumo o contextos recreativos específicos. La hipótesis es que diferentes prácticas y contextos se asocian a perfiles diferenciales de consumo de sustancias. A favor de esta hipótesis, el consumo de estimulantes (FernándezCalderón et al., 2013) y marihuana (Bonino, Pautassi \& Pilatti, 2015) es mayor entre los asistentes a fiestas electrónicas y recitales de rock, respectivamente, que en población general del mismo rango etario (Pilatti, Bonino, Ensinck Atienza, Rivarola Montejano, \& Pautassi, 2015). Los resultados del presente trabajo indican un efecto contundente y sustantivo de la asistencia a previas sobre el número de consecuencias relacionadas con el alcohol.

Los resultados indicaron similar ocurrencia de CEEA entre hombres y mujeres, lo que es consistente con estudios que indican un efecto cada vez más despreciable del sexo sobre los patrones de consumo de alcohol (Grucza, Norberg, \& Bierut, 2009; Keyes, Li, \& Hasin, 2011). Asimismo, vimos un efecto facilitador de la edad de inicio del consumo sobre la frecuencia de asistencia a previas y sobre el consumo en las mismas. Una posibilidad es que el inicio temprano incremente el consumo de alcohol y de consecuencias negativas vía una mayor asistencia a previas. La exposición a modelos sociales de consumo incrementa las conductas de consumo de alcohol (Courtney \& Polich, 2009). Posiblemente, los bebedores tempranos están también más tempranamente expuestos a los modelos de consumo de las previas, cuyo correlato es una modalidad excesiva de consumo de alcohol. Otra posibilidad es que los bebedores tempranos, que se caracterizan por exhibir modalidades de consumo excesivo de alcohol, se sientan atraídos por este tipo de contexto de consumo.

Una limitación del trabajo es que no se preguntó cuánto alcohol se consumió después de la previa y los reportes de semana usual e intensa no diferenciaron en consumo por dentro y por fuera de previa. Otras limitaciones son el diseño transversal (que impide establecer relaciones de causalidad) y aspectos relacionados a la formación de la muestra. Respecto a este último punto, la muestra estuvo limitada a aquellos jóvenes con acceso a internet. 
Asimismo, la mayoría de los participantes eran estudiantes universitarios lo que, según el último censo poblacional realizado en Argentina (2010), se asocia a un mayor nivel socio-económico. Otro aspecto a considerar con este tipo de recolección de datos (i.e., encuesta online) es la potencial duplicación de participación, especialmente cuando hay premios.

Más allá de estas limitaciones, el trabajo aporta información que describe las conductas de consumo de alcohol en jóvenes asistentes a previas y, además, sugiere que realizar este tipo de prácticas de consumo de alcohol sería en sí mismo un factor de riesgo para el involucramiento en trayectorias de consumo problemático de alcohol. Los resultados también desacreditan la extendida noción popular de que, al menos ocasionalmente, puede ser adecuado aprobar la realización de previa por parte de padres o cuidadores, a fin de que el consumo de alcohol ocurra en el contexto aparentemente seguro o protegido del hogar.

\section{REFERENCIAS}

Bachrach, R. L., Merrill, J. E., Bytschkow, K. M., \& Read, J. P. (2012). Development and initial validation of a measure of motives for pregaming in college students. Addict Behav, 37(9), 1038-1045. doi: 10.1016/j.addbeh.2012.04.013

Barnett, N. P., Orchowski, L. M., Read, J. P., \& Kahler, C. W. (2013). Predictors and consequences of pregaming using day- and weeklevel measurements. Psychol Addict Behav, 27(4), 921-933. doi: $10.1037 / \mathrm{a} 0031402$

Barry, A. E., Stellefson, M. L., Piazza-Gardner, A. K., Chaney, B. H., \& Dodd, V. (2013). The impact of pregaming on subsequent blood alcohol concentrations: an event-level analysis. Addict Behav, 38(8), 23742377. doi: 10.1016/j.addbeh.2013.03.014

Bonino, P., Pautassi, R.M., Pilatti, A. (2015). Perfiles de consumo de sustancias en jovenes asistentes a recitales de rock. Trabajo presentado en la XV Reunión Nacional y IV Encuentro Internacional de la Asociación Argentina de las Ciencias del Comportamiento. San Miguel de Tucumán, Argentina.

Borsari, B., Boyle, K. E., Hustad, J. T., Barnett, N. P., O'Leary Tevyaw, T., \& Kahler, C. W. (2007). Drinking before drinking: pregaming and drinking games in mandated students. Addict Behav, 32(11), 26942705. doi: 10.1016/j.addbeh.2007.05.003

Courtney, K. E., \& Polich, J. (2009). Binge drinking in young adults: Data, definitions, and determinants. Psychol Bull, 135(1), 142-156. doi: $10.1037 / a 0014414$

Dawson, D. (2003). Methodological issues in measuring alcohol use. Alcohol research \&
Del Zotto Libonati, P. (2015). Consumo Episódico Intensivo de Alcohol en jóvenes argentinos durante la realización de la Previa. Tesis de Doctorado. Universidad de Valencia, España.

Fernández-Calderón, F, Lozano-Rojas, Ó.M., Rojas-Tejada, A.J. (2013). Raves y consumo de drogas desde una perspectiva epidemiológica y psicosocial: una revisión bibliográfica sistemática. Adicciones 25 , 269-279.

Finlay, A. K., Ram, N., Maggs, J. L., \& Caldwell, L. L. (2012). Leisure Activities, the Social Weekend, and Alcohol Use: Evidence From a Daily Study of First-Year College Students. J Stud Alcohol Drugs 73, 250-259.

Foster, J. H., \& Ferguson, C. (2014). Alcohol 'pre-loading': a review of the literature. Alcohol Alcohol, 49(2), 213-226. doi: 10.1093/alcalc/agt135

Grucza, R. A., Norberg, K. E., \& Bierut, L. J. (2009). Binge drinking among youths and young adults in the United States: 1979-2006. J Am Acad Child Adolesc Psychiatry, 48(7), 692-702. doi: 10.1097/CHI.0b013e3181a2b32f

Haas, A. L., Smith, S. K., Kagan, K., \& Jacob, T. (2012). Pre-college pregaming: practices, risk factors, and relationship to other indices of problematic drinking during the transition from high school to college. Psychol Addict Behav 26(4), 931-938. doi: 10.1037/a0029765

Health 27, 18-29.

Hingson, R. W., Heeren, T., Winter, M. \& Wechsler, H. (2005). Magnitude of alcohol-related mortality and morbidity among U.S college students ages 18-24: changes from 1998 to 2001 . Annual reviews. 26: 259-79.

Hustad, J. T., Mastroleo, N. R., Urwin, R., Zeman, S., LaSalle, L., \& Borsari, B. (2014). Tailgating and pregaming by college students with alcohol offenses: patterns of alcohol use and beliefs. Subst Use Misuse, 49(14), 1928-1933. doi: 10.3109/10826084.2014.949008

Kenney, S. R., Hummer, J. F., \& Labrie, J. W. (2010). An examination of pregaming and drinking game playing during high school and their impact on alcohol-related risk upon entrance into college. J Youth Adolesc, 39(9), 999-1011. doi: 10.1007/s10964-009-9473-1

Keough, M. T., O'Connor, R. M., \& Read, J. P. (2016). Replication and Validation of the Young Adult Alcohol Consequences Questionnaire in a Large Sample of Canadian Undergraduates. Alcohol Clin Exp Res, 40(5), 1093-1099. doi: 10.1111/acer.13039

Keyes, K. M., Li, G., \& Hasin, D. S. (2011). Birth cohort effects and gender differences in alcohol epidemiology: a review and synthesis. Alcohol Clin Exp Res, 35(12), 2101-2112. doi: 10.1111/j.15300277.2011.01562.x

LaBrie, J. W., Earle, A. M., Hummer, J. F., \& Boyle, S. C. (2016). Is Prepartying a Cause of Heavy Drinking and Consequences Rather Than Just a Correlate? A Longitudinal Look at the Relationship Between Prepartying, Alcohol Approval, and Subsequent Drinking and Consequences. Subst Use Misuse, 51(8), 1013-1023. doi: 10.3109/10826084.2016.1152493

LaBrie, J. W., Hummer, J. F., Pedersen, E. R., Lac, A., \& Chithambo, T. (2012). Measuring college students' motives behind pregaming drinking: development and validation of the pregaming motivations 
inventory. Addict Behav, 37(8), 962-969. doi: 10.1016/j.addbeh.2012.04.003

Lee, L. O., Young-Wolff, K. C., Kendler, K. S., \& Prescott, C. A. (2012). The effects of age at drinking onset and stressful life events on alcohol use in adulthood: a replication and extension using a populationbased twin sample. Alcohol. Clin. Exp. Res. 36, 693-704. doi: 10.1111/j.1530-0277.2011.01630.x

Merrill, J. E., Vermont, L. N., Bachrach, R. L., \& Read, J. P. (2013). Is the pregame to blame? Event-level associations between pregaming and alcohol-related consequences. J Stud Alcohol Drugs, 74(5), 757764.

Miller, M. B., Borsari, B., Fernandez, A. C., Yurasek, A. M., \& Hustad, J. T. (2016). Drinking Location and Pregaming as Predictors of Alcohol Intoxication Among Mandated College Students. Subst Use Misuse, 51(8), 983-992. doi: 10.3109/10826084.2016.1152496

National Institute on Alcohol Abuse and Alcoholism [NIAAA] (2004). National Institute of Alcohol Abuse and Alcoholism Council approves definition of binge drinking. NIAAA Newsletter N3. http://pubs.niaaa.nih.gov/publications/Newsletter/winter2004/Ne wsletter_Number3.htm

Pan-American Health Organization [PAHO] (2015). Informe sobre la situación regional sobre el alcohol y la salud en las Américas. Washington, DC: Organización Panamericana de la Salud, Contract No.: 978-92-75-31855-3.

Paves, A. P., Pedersen, E. R., Hummer, J. F., \& Labrie, J. W. (2012). Prevalence, social contexts, and risks for pregaming among ethnically diverse college students. Addict Behav, 37(7), 803-810. doi: 10.1016/j.addbeh.2012.03.003

Pedersen, E. R., \& Labrie, J. (2007). Partying before the party: examining pregaming behavior among college students. J Am Coll Health, 56(3), 237-245. doi: 10.3200/JACH.56.3.237-246

Pilatti A, Fernández Calderón F, Bonino P, Ensinck Atienza G, Rivarola Montejano, G, \& Pautassi, R (2015). Effect of age of drinking onset on patterns of alcohol, tobacco, marihuana, stimulants and hallucinogens use, during young adulthood. Paper presented at the European Society for Biomedical Research on Alcoholism (ESBRA) Congress. Valencia, España.

Pilatti, A., Caneto, F., Garimaldi, J.A., Vera Bdel, V., \& Pautassi, R.M. (2014). Contribution of time of drinking onset and family history of alcohol problems in alcohol and drug use behaviors in Argentinean college students. Alcohol Alcohol 49, 128-137.

Pilatti, A., Godoy, J.C., Brussino, S.A., Pautassi, R.M. (2013). Patterns of substance use among Argentinean adolescents and analysis of the effect of age at first alcohol use on substance use behaviors. Addict Behav, 2847-2850.

Pilatti, A., Read, J.P., \& Caneto, F. (2016). Validation of the Spanish Version of the Young Adult Alcohol Consequences Questionnaire (S-YAACQ). Psychol Assess 28, e49-61.

Read, J. P., Beattie, M., Chamberlain, R., \& Merrill, J. E. (2008). Beyond the "Binge" threshold: heavy drinking patterns and their association with alcohol involvement indices in college students. Addict Behav 33(2), 225-234. doi: 10.1016/j.addbeh.2007.09.001

Read, J. P., Kahler, C. W., Strong, D. R., \& Colder, C. R. (2006). Development and preliminary validation of the young adult alcohol consequences questionnaire. J Stud Alcohol, 67(1), 169-177.

Rutledge, P. C., Bestrashniy, J. R., \& Nelson, T. F. (2016). Problematic Drinking Among Postgraduate Students: Binge Drinking, Pregaming, and Mixing Alcohol With Energy Drinks. Subst Use Misuse, 51(8), 972-982. doi: 10.3109/10826084.2016.1152499

Squeglia, L. M., Jacobus, J., \& Tapert, S. F. (2009). The influence of substance use on adolescent brain development. Clin EEG Neurosci 40, 31-38.

Vetreno, R. P., Yaxley, R., Paniagua, B., Johnson, G. A., \& Crews, F. T. (2017). Adult rat cortical thickness changes across age and following adolescent intermittent ethanol treatment. Addiction Biology 22, 712-723 doi: 10.1111/adb.12364

Wechsler, H., Davenport, A., Dowdall, G., Moeykens, B., \& Castillo, S. (1994). Health and behavioral consequences of binge drinking in college. A national survey of students at 140 campuses. Journal of the American Medical Association 272, 1672-7.

White, A. \& Hingson, R. (2014). The Burden of Alcohol Use: Excessive Alcohol Consumption and Related Consequences Among College Students. Alcohol Research: Current Reviews, 35, 201-218.

Zamboanga, B. L., Borsari, B., Ham, L. S., Olthuis, J. V., Van Tyne, K., \& Casner, H. G. (2011). Pregaming in high school students: relevance to risky drinking practices, alcohol cognitions, and the socia drinking context. Psychol Addict Behav, 25(2), 340-345. doi: 10.1037/a0022252 